\title{
RasGEF1A and RasGEF1B are guanine nucleotide exchange factors that discriminate between Rap GTP-binding proteins and mediate Rap2-specific nucleotide exchange
}

\author{
Elif Yaman ${ }^{1, *}$, Raphael Gasper ${ }^{2} *{ }^{*}$, Carolin Koerner ${ }^{2}$, Alfred Wittinghofer ${ }^{2}$ and Uygar H. Tazebay ${ }^{1}$ \\ 1 Department of Molecular Biology and Genetics, Bilkent University, Ankara, Turkey \\ 2 Abteilung Strukturelle Biologie, Max-Planck-Institut für Molekulare Physiologie, Dortmund, Germany
}

\section{Keywords}

G-proteins; guanine nucleotide exchange;

Rap2; Ras family; RasGEF1

\section{Correspondence}

U. H. Tazebay, Department of Molecular Biology and Genetics, Bilkent University, 06800 Bilkent Ankara, Turkey

Fax: +903122665097

Tel: +903122902419

E-mail: tazebay@fen.bilkent.edu.tr

*These authors contributed equally to this work

(Received 28 April 2009, revised 18 June 2009, accepted 22 June 2009)

doi:10.1111/j.1742-4658.2009.07166.x
The highly conserved RasGEF1 family of proteins contain a C-terminal CDC25-Ras exchange motif domain and an N-terminal RasGEF-N domain, and are of unknown function and specificity. Using purified RasGEF1A and RasGEF1B proteins, as well as Ras family proteins, we established that RasGEF1A and RasGEF1B function as very specific exchange factors for Rap2, a member of the Rap subfamily of Ras-like G-proteins. They do not act on Rap1 or other members of the Ras subfamily. Although Rap2 was implicated in the regulation of cell adhesion, the establishment of cell morphology, and the modulation of synapses in neurons, no specific guanine nucleotide exchange factor for Rap2 was previously identified. Using reciprocal site-directed mutagenesis, we analyzed residues that allow RasGEF1 proteins to discriminate between Rap1 and Rap2, and we were able to identify Phe39 in the switch I region of Rap2 as a specificity residue. Mutation of the corresponding Ser39 in Rap1 changed the specificity and allowed the nucleotide exchange of Rap1(S39F) to be stimulated by RasGEF1B.

\section{Introduction}

Rap proteins are members of the Ras family of proteins, which function as nucleotide-driven switches that cycle between inactive GDP-bound and active GTPbound conformations, thereby coupling cell surface receptor activation to downstream effector pathways. Just as with other members of the superfamily, activation is regulated by guanine nucleotide exchange factors (GEFs), which mediate replacement of GDP with GTP, and by GTPase-activating proteins, which increase the intrinsic hydrolysis reaction by several orders of magnitude [1-3].

Rap proteins are involved in a variety of cellular processes. In lymphocytes, they regulate integrin-mediated cell adhesion, cytoskeletal rearrangements, cell migration, and cadherin-mediated cell junction formation [4-6], whereas in neuronal cells they modulate axonal differentiation, dendritic development, spine morphology, and synaptic plasticity [7-9]. Rap family members are considered to be close homologs of Ras proteins, and their effector-interacting regions are very similar [10]. This gives them the ability to interact with Ras effectors such as Raf-1, B-Raf, RalGDS, Rgl, Rlf, AF6, RasSF1, and phospholipase-CE [11-14], and, depending on the cellular context and extracellular stimuli, they may interfere with Ras signaling. However, it is more likely that they exert an entirely Ras-independent specific function by using a subset of potential Ras effectors [15-19].

\section{Abbreviations}

GEF, guanine nucleotide exchange factor; GSH, glutathione; GST, glutathione-S-transferase; IPTG, isopropyl thio- $\beta$-D-galactoside; mant-GNP, $\mathrm{N}$-methylanthranil acid-labeled guanine nucleotide; REM, Ras exchange motif. 
On the basis of the sequence homology, the Rap family is divided into two subgroups, Rap1 and Rap2, with a total of five members: Rap1A, Rap1B, Rap2A, Rap2B, and Rap2C [20]. No functional differences have been reported between the members of each group. There is about $95 \%$ sequence identity between Rap1A and Rap1B, and about 90\% identity between Rap2A, Rap2B, and Rap2C. Effector-binding regions, including switch I, show the highest conservation, and C-terminal regions the lowest. Similarly, Rap1 proteins are closely related (about $70 \%$ identical) to Rap2 subfamily members. Although identical or overlapping functions have been described for Rap1 and Rap2 proteins $[4,21,22]$, a number of studies showing functional distinctions and different effector-protein interactions have been reported for Rap1 and Rap2 proteins [7,8,23,24]. Recently, specific Rap2-binding proteins such as RPIP8, TNIK and MAP4K4 were reported as candidate effectors [19,25,26]; however, their cellular functions as Rap2 effectors are not fully established.

The SMART database (http://smart.embl-heidelberg.de/smart) currently lists 28 RasGEF-CDC25 domains, most of which, if not all, occur in tandem with an N-terminal extension RasGEF-N-Ras exchange motif (REM) domain and which, according to structural analysis, are in close contact with each other [27,28]. Of these, some have been identified as activators specific for Ras, Rap or Ral. Despite established differences in cellular function and subcellular localization [29-31] of Rap1 and Rap2, none of the currently known Rap-activating GEFs with the REMCDC25 domain tandem were shown to have specificity for Rap2. The major classes of GEF proteins with specificities for Rap GTP-binding proteins include C3G, Epac1, Epac2, PDZ-GEFs, and RapGRPs [32]. $\mathrm{C} 3 \mathrm{G}$ is specific to Rap1, and controls its tyrosine kinase-induced activation [33]. Other classes of RapGEFs are common for both Rap1 and Rap2. For instance, members of the Epac family of GEFs (Epac1 and Epac2) are directly regulated by cAMP, and they catalyze nucleotide exchange of Rap in response to this cyclic nucleotide [34,35]. Members of the PDZ-GEF family have dual specificities for Rap1 and Rap2, and they are proposed to regulate contact-induced activation of Rap at cell junctions [32,36]. On the other hand, RasGRP family GEFs (also known as CalDAGGEFs) have broader specificity, and they activate Rap, Ras, or both, in response to diacylglycerol and/or $\mathrm{Ca}^{2}+[2,37,38]$.

In the present work, we have studied the GEF activities of two members of a previously uncharacterized family of RasGEF proteins, RasGEF1A and RasGEF1B, and we show here that these novel factors function as Rap2-specific GEFs. They do not affect nucleotide exchange of either Rap1 or other tested members of the Ras subfamily. Further characterization of RasGEF1-Rap interactions by comparative mutational studies revealed that a single residue (Phe39) in the switch I region of Rap2A determines the specificity of its functional interaction with the RasGEF1 family of exchange factors. This is the first description of GEFs specific for Rap2.

\section{Results}

In order to investigate possible GEF functions of RasGEF1A and RasGEF1B, two putative nucleotide exchange factors of the CDC25-REM RasGEF family, the full-size proteins were expressed as glutathione$S$-transferase (GST) fusions in Escherichia coli and purified by using glutathione-agarose beads (Experimental procedures). Nucleotide exchange activities of these two RasGEF1 proteins were assessed for using the fluorescence assay established by us [39]. G-proteins were loaded with fluorescent mant-nucleotide and incubated with RasGEF1A and RasGEF1B in the presence of a large excess of unlabeled nucleotide. As it has previously been shown that the stimulation of nucleotide dissociation by GEFs is independent of the nature of the nucleotide [39-41], for reasons of stability and convenience we

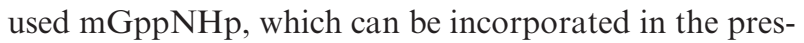
ence of alkaline phosphatase. The release of fluorescent nucleotide was followed as a decrease in fluorescence in real time and was fitted to a single exponential (Table S1). To identify the specificity of RasGEF proteins, we used various G-proteins from the Ras subfamily, such as H-Ras, R-Ras, M-Ras, TC21, Di-Ras2, Rheb, RERG, RalB, Rap1A, Rap1B, and Rap2A [42-44]. Representative fluorescence traces for the RasGEF1A and RasGEF1B reactions are shown in Fig. 1A,B and the experiments are summarized in Fig. 1C (see also Table S1).

As expected, the intrinsic dissociation rates of the G-proteins varied remarkably, with Di-Ras2 and RERG having rates as high as $1.92 \times 10^{-3} \cdot \mathrm{s}^{-1}$ and $5.49 \times 10^{-3} \cdot \mathrm{s}^{-1}$, respectively. Regardless of the protein concentrations used in the reactions, RasGEF1A or RasGEF1B did not significantly stimulate nucleotide exchange on most tested Ras family members, such as H-Ras, R-Ras, M-Ras, TC21, Di-Ras2, Rheb, RERG, or RalB (Fig. 1). However, both RasGEF1A and RasGEF1B stimulated exchange on Rap2A, whereas they had no GEF activities on the other members of the Rap subfamily, Rap1A or Rap1B, demonstrating that the RasGEF1 family members show a similar specificity (Fig. 1A,B). Quantitatively, however, the rate of 


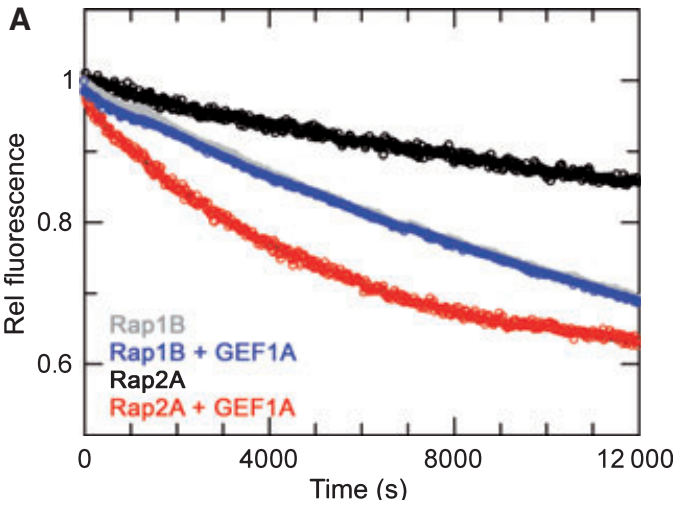

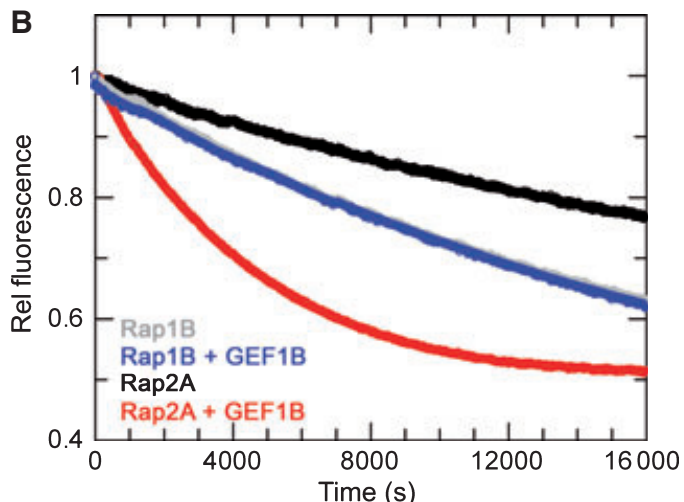

C

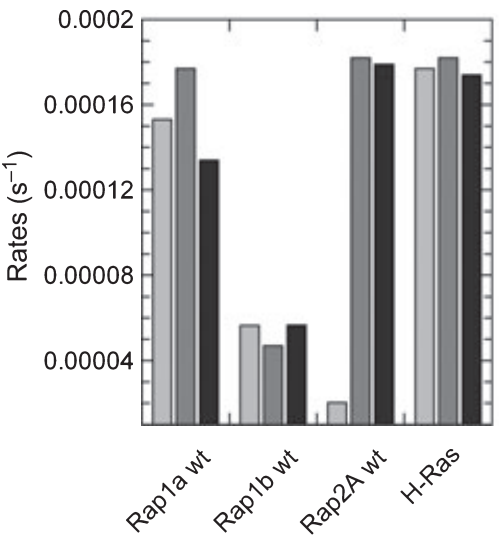

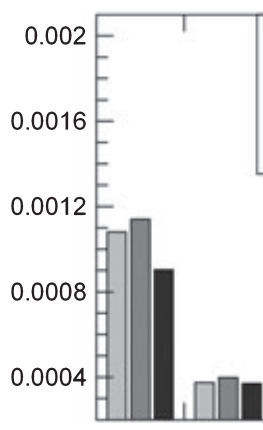

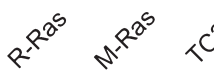
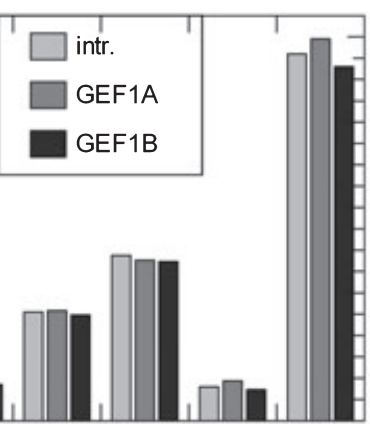

$\beta^{20} \quad \nabla^{e^{0}}$

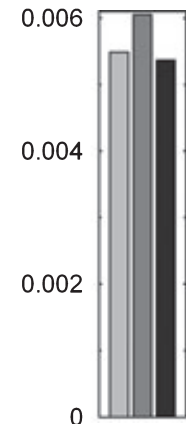

से

Fig. 1. Guanine nucleotide exchange rates of Ras family of G-proteins in the presence of RasGEF1A and RasGEF1B. G-proteins indicated in the figures or below each set of bar graphs were loaded with mant-GNP, and dissociation of the fluorescent nucleotide in the absence (intrinsic rate) or in the presence of $200 \mathrm{nM}$ RasGEF1A or $4 \mu \mathrm{M}$ RasGEF1B were followed. Values were obtained from at least three independent experiments, and representative graphs are displayed. GEF1A and GEF1B stand for RasGEF1A and RasGEF1B, respectively. (A, B) Guanine nucleotide exchange reaction of $150 \mathrm{~nm}$ Rap2A and Rap1B with $200 \mathrm{~nm}$ RasGEF1A (A) and with $4 \mu \mathrm{M}$ RasGEF1B (B). Different experimental conditions are indicated by colored lines. (C) Stimulation of the Ras family of G-proteins by RasGEF1A or RasGEF1B in the presence of exchange factors at the concentrations indicated above. intr., intrinsic exchange rates of G-proteins.

nucleotide release from Rap2A in the presence of $200 \mathrm{~nm}$ RasGEF1A $\left(1.82 \times 10^{-4} \cdot \mathrm{s}^{-1}\right)$ was only comparable to the rate of release stimulated by a 20 -fold higher concentration of RasGEF1B $\left(1.79 \times 10^{-4} \cdot \mathrm{s}^{-1}\right.$ for $4 \mu \mathrm{M}$ exchange factor), indicating a higher activity of RasGEF1A than of RasGEF1B. Altogether, these results clearly indicated that both RasGEF1A and RasGEF1B belong to a novel class of RapGEFs that are specific for Rap2.

Rap1A, Rap1B and Rap2A are closely related proteins. Their switch I region (amino acids 25-40) and switch II region (amino acids 57-75), both of which were previously shown to contain critical residues for G-protein-GEF interactions [27,28,33], are nearly identical. In an effort to identify residues that are critical for the specificity of RasGEF1A and RasGEF1B interactions with Rap2, we first compared the switch I and switch II regions of Rap proteins and identified residues that are localized in these conserved func- tional domains, but differ between the two G-proteins (Fig. 2A). In parallel, by taking advantage of a previously solved structure of Rap1B in complex with Epac2 [27], we prepared a structural model in order to detect residues in Rap2A that are distinct from their counterparts in Rap1A and Rap1B and are expected to interact with RasGEF1s (Fig. 2B). The predominant part of the interface between Rap2A and RasGEF1B includes conserved regions of the GEF protein, with the exception of two regions that are in close contact with the G-protein but do not show sequence conservation (Fig. 2B). The first region includes the interface for binding to the switch I region of Rap $2 \mathrm{~A}$, and the second is located around helix $\alpha 2$ of Rap, C-terminal of the switch II region (Fig. 2B). The contact area around the switch II region of Rap is conserved between RasGEF1B and Epac2. The only switch I residue that is not conserved between Rap1 and Rap2 is Phe39 (Rap2A), which corresponds to Ser39 in 

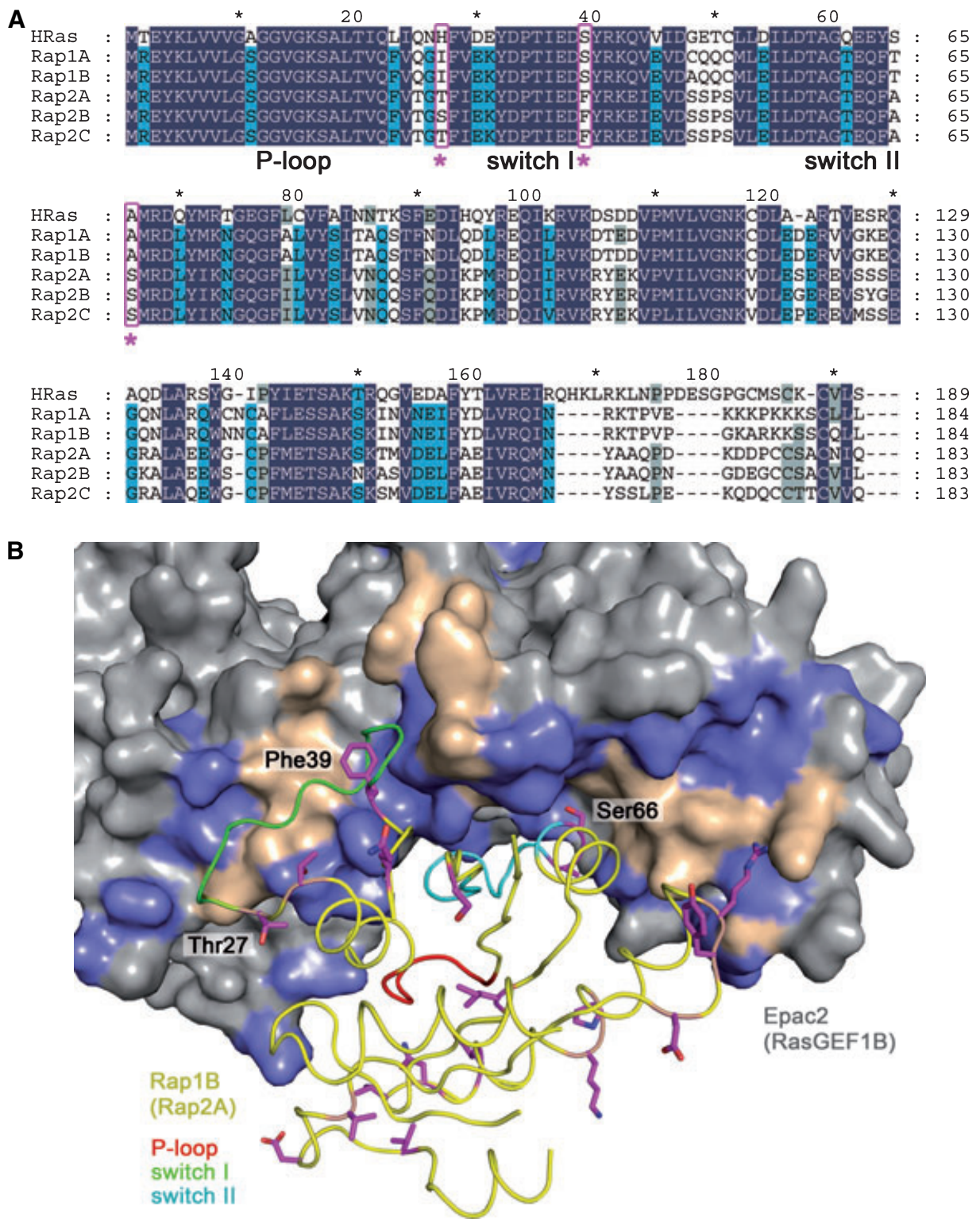

Fig. 2. Positions of residues selected for mutational analysis. (A) Sequence alignment of H-Ras, Rap1A, Rap1B, Rap2A, Rap2B and Rap2C. Residues that have $100 \%, 80 \%$ and $60 \%$ similarity are in dark blue, blue and gray, respectively. Thr27, Phe39, and Ser66, chosen for mutational analysis, are indicated by blue boxes and an asterisk below. (B) Predictive model of a Rap2A-RasGEF1B complex. The sequence of RasGEF1B was overlain with Epac2 from the Rap1B-Epac2-CAMP complex (Protein Data Bank code: 3CF6 [27]), using MODELLER software [57], remodeling flexible loops and sequence differences caused by gaps in sequence alignment. Residues that were different between Rap1B and Rap2A were mutated in Rap1B-Epac2-cAMP by using coot software [58]. Sequence differences between Rap2A and Rap1B are highlighted in magenta; the secondary structure of Rap1B is in yellow. Switch I (green), switch II (cyan) and P-loop (red) regions are indicated. RasGEF1B is shown as a gray surface with conserved residues (as compared with Epac2) in blue, and interface residues that are not conserved in light brown.

Rap1B (Fig. 2A). The side chain of Ser39 is encircled by the switch I region (Fig. 2B) suggesting that Phe39 of Rap2 will cause an entirely different conformation of this loop region. Interestingly, this region is not conserved between RasGEF1B and Epac2. Helix $\alpha 2$ of Rap is in direct contact with an $\alpha$-helix in Epac2 that is remodeling the switch II region for the GEF reaction. This interaction mostly depends on the main chain of Epac2, and the only side chain interaction is established by Ser66 (Rap2A), which is an Ala (Ala66) in Rap1B (Fig. 2A,B). Therefore, Phe39 and Ser66 in Rap2 were identified as possible discriminatory 
candidates for the G-protein-GEF interaction. A third possible candidate was Thr27 in Rap2A, as this hydrophilic residue was replaced with a hydrophobic Ile in Rap1B, and structural data indicated this residue to be in contact with a conserved region of Epac2 (Fig. 2B). We then carried out a comparative mutational analysis of the interaction between RasGEF1 and Rap proteins by converting Thr27, Phe39 and Ser66 in Rap2 to the residues found at the same positions in Rap1. Rap2 proteins carrying T27I, F39S and S66A mutations were expressed, and nucleotide exchange assays were carried out in the presence of RasGEF1A and RasGEF1B as described for the wild-type proteins (Fig. 3). Mutating Ser66 in the switch II region of Rap2 did not affect exchange rates by RasGEF1s (Fig. 3A,B), even though this region was previously shown to contain residues important for G-proteinGEF interactions [45-47]. Mutation T27I in the switch I region of Rap2 slowed down the exchange in the presence of RasGEF1s (Fig. 3A,B) but the effect was also small. Clearly, the most dramatic effect was obtained by mutating residue 39 of Rap2, which completely abolished nucleotide exchange of Rap2(F39S) catalyzed by RasGEF1A or RasGEF1B (Fig. 3A,B), indicating that Phe39 is essential for the catalytic interaction of Rap2 with RasGEF1 family members.

In order to assess whether simply converting the residue at the same position in Rapl to Phe (as in Rap2A) could lead to the stimulation of exchange by RasGEF1 family members, we mutated Ser39 in Rap1, and tested the nucleotide exchange activities of RasGEF1s on mutant Rap1(S39F). Interestingly, we observed that even though having Phe instead of Ser at position 39 of Rap1 was not sufficient for RasGEF1A to stimulate nucleotide exchange of Rap1(S39F) (Fig. 3C), RasGEF1B could stimulate the exchange of mutant Rap1(S39F), albeit at a slower rate (Fig. 3D) than that of Rap2 (Fig. 1). Importantly,
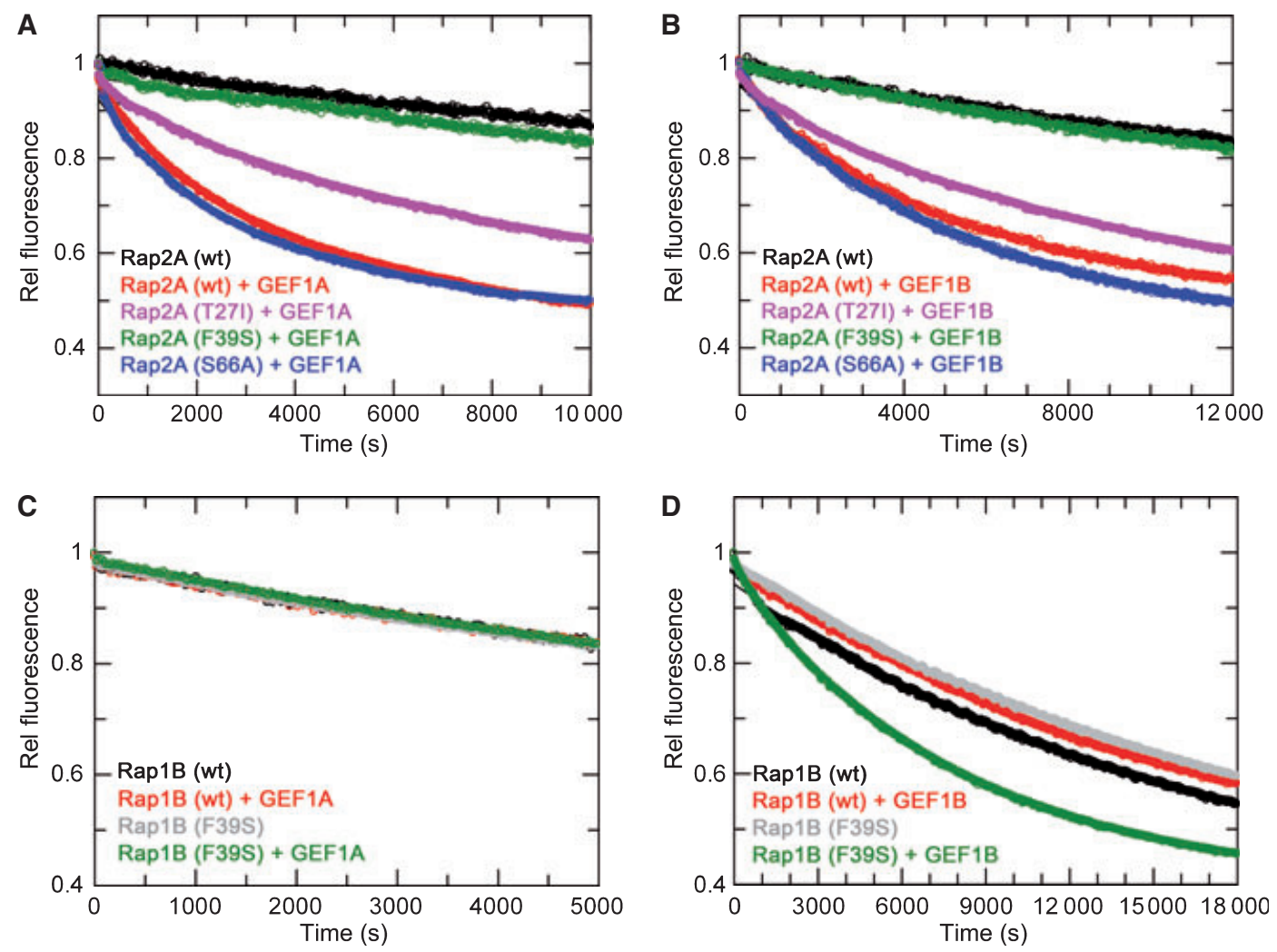

Fig. 3. Guanine nucleotide exchange rates of wild-type and mutant Rap proteins. Wild-type or mutant Rap proteins were loaded with fluorescent mant-GNP, and the release of nucleotide was measured in real time in the absence or presence of GEFs. Values were obtained from at least three independent experiments, and representative graphs are displayed. GEF1A and GEF1B stand for RasGEF1A and RasGEF1B, respectively. (A) Guanine nucleotide exchange reaction of $150 \mathrm{~nm}$ wild-type Rap2A, Rap2A(F39S), Rap2A(S66A) and Rap2A(T27l) with 200 nM RasGEF1A. (B) Guanine nucleotide exchange reaction of $150 \mathrm{~nm}$ wild-type Rap2A, Rap2A(F39S), Rap2A(S66A) and Rap2A(T27l) with $4 \mu \mathrm{M}$ RasGEF1B. (C) Guanine nucleotide exchange reaction of $150 \mathrm{~nm}$ wild-type Rap1B and Rap1B(S39F) with $200 \mathrm{~nm}$ RasGEF1A. (D) A similar exchange reaction as in $(C)$, with $4 \mu \mathrm{M}$ RasGEF1B. Different experimental conditions are indicated by colored lines. 
these results indicate that Phe39 is not only essential for stimulation of Rap2 by the RasGEF1 family of exchange factors, but is also sufficient, at least partially, for the specificity between Rap1 and Rap2 and for RasGEF1B to act as a cognate GEF on Rap1.

\section{Discussion}

Previous studies focusing on the cellular functions of Rap proteins have revealed that they could function independently of Ras. Rapl has been studied extensively in many different cell types and organisms, and it has critical roles in cytoskeletal rearrangement, integrin-mediated cell adhesion, platelet activation, and cadherin-mediated cell junction formation [10,35]. Even though the expression patterns of Rap1 and Rap2 overlap, much less is known about a particular function of Rap2, indicating that the two subgroups (Rap1 and Rap2) could have different functions and be differentially regulated $[7,8,48]$. Supporting the notion of different functions, it has been shown that Rap2 is not involved in integrin activation but rather in Wnt- $\beta$-catenin signaling during dorsoventral patterning in Xenopus development [49], and that Rap2, but not Rap1, is expressed in red blood cells [50].

In this study, we screened a large set of small GTPbinding proteins of the Ras subfamily for stimulation of nucleotide exchange by two previously uncharacterized putative GEFs of the Cdc25 family, RasGEF1A and RasGEF1B. Both factors stimulated nucleotide exchange of only Rap2A, and did not have any effect at all on other distantly or closely related members of the Ras family (Fig. 1A-C). Comparison of exchange reaction rates of RasGEF1A and RasGEF1B suggested that RasGEF1A leads to about 20-fold faster release of nucleotide from Rap2A than that seen in the presence of RasGEF1B (Figs 1 and S1). We noticed that the nucleotide exchange rate of RasGEF1B is also slower than that of other RapGEFs, such as Epac or C3G [51]. RasGEF1A and RasGEF1B are comparatively small GEFs, without any apparent additional domain that could potentially be autoinhibitory. However, weak affinities between GEFs and their cognate G-proteins have been noticed previously, and could indicate that, in vivo, RasGEF1 activity is substantially increased by translocation to the plasma or internal membranes, which increases the local concentration of substrate and enzyme.

A multiple human tissue northern blot analysis carried out by Ura et al. [52] revealed that brain and spinal cord are two tissues with very strong RasGEF1A expression. In contrast to our data, these authors reported that RasGEF1A could act as a GEF for
H-Ras, K-Ras, and N-Ras, but sufficient experimental details were not given, and nor were other Ras proteins tested in comparison. In our hands, when unfused Ras proteins were used rather than GST-linked proteins, and phosphocellulose filter binding was avoided, RasGEF1A has no effect on the nucleotide exchange of classic Ras proteins in the solution assay. The gene encoding murine RasGEF1B was first identified in a study reporting upregulation of its expression in macrophages in response to mucin-like glycoproteins of a protozoan parasite, Trypanosoma cruzi, and it was initially named GPI $\gamma 4$ [53]. A study on tissue-specific expression of RasGEF1B homolog in developing zebrafish embryos indicated that this gene is also strongly expressed in midbrain and hindbrain tissues [54]. Interestingly, specific functions of Rap2 were identified in different neuronal processes, such as synaptic depotentiation and modulation of neuronal morphology [7-9,48], and it is possible that the two RasGEF1 family members play critical roles in the spatial and temporal regulation of Rap2 activity at different rates in hippocampal neurons during the control of excitatory synapses.

Although the structural model of the RasGEF1Rap2 complex suggested Ser66 in the switch II region to be located in or close to the interface, the S66A mutation in Rap2A did not affect nucleotide exchange by RasGEF1 family members, suggesting that the mild Ser $\rightarrow$ Ala substitution can easily be accommodated in the interface. This is in line with the structure of the Epac-Rap1 complex, where Ala66 makes a main chain water-mediated contact with Asn803 of Epac [27]. Whereas wild-type Rap2 is not stimulated by $\mathrm{C} 3 \mathrm{G}$, the double-mutated (T65A/S66A) Rap2 can be partially stimulated by $\mathrm{C} 3 \mathrm{G}$, just as a more extensive quadruple mutation of the switch II region made Ras partially responsive to $\mathrm{C} 3 \mathrm{G}$ [33]. All of this supports the notion that the switch II region is involved in binding and specificity, and that the interface between Cdc25-GEFs and their cognate G-proteins shows great plasticity $[27,28]$.

Conversion of Thr27 in switch I of Rap2A to Ile (as in Rap1) reduces the nucleotide exchange rate (Fig. 3A,B), indicating a functional role for this residue. The most dramatic effect is, however, due to the F39S mutation in Rap2A, which completely abolished nucleotide exchange activity by the two cognate GEFs, RasGEF1A and RasGEF1B (Fig. 3A,B). Residue 39 is located in the switch I region previously shown to be critical in Ras-Cdc25, Rap-Epac and Rap1-C3G interactions [27,28,33]. Furthermore, it was previously shown that mutations at Thr35 and Glu37 positioned in the switch I region of Rap1 decreased its interaction with $\mathrm{C} 3 \mathrm{G}$ by a factor of four [33]. These previous data are in agreement with our results regarding the critical 
role of the switch I region and, in particular, Phe39 in the interaction of Rap2A with RasGEF1A and RasGEF1B. Phe39 is also a specificity determinant, as the S39F mutation in Rap1B allows RasGEF1B to act on Rap1B. As RasGEF1A does not have the same stimulatory effect on Rap1(S39F), regardless of its concentration (from $200 \mathrm{~nm}$ up to $20 \mu \mathrm{M}$; Fig. S1), this suggests that the two GEFs establish different contacts even though they are $61 \%$ identical.

These results indicate that even a single amino acid difference in the switch I region of Rap GTP-binding proteins may determine the specificities of interactions with cognate nucleotide exchange factors. In fact, in a previous study, residue 39 was also shown to determine the specificity of interaction of Rap2 with the downstream effector RAPL/NORE [6]. The Phe39 $\rightarrow$ Ser39 sequence difference in the switch I region of Rap1 and Rap2 is thus a major determinant of the activation of different Rap group members and their downstream biological effects.

\section{Experimental procedures}

\section{Plasmid constructs}

cDNAs corresponding to the RasGEF1A and RasGEF1B genes were cloned into the pGEX-4T-1 expression vector and transformed into E. coli BL21 CodonPlus RIL cells. DiRas2, M-Ras, R-Ras, RalB, TC21, RERG and Rheb were cloned into pGex4T1-TEV and expressed in E. coli BL21 DE3 cells. C-terminally truncated forms of H-Ras, Rap1A, Rap1B and Rap2A were cloned into ptac vectors and expressed in CK600K cells. pGEX-4T-3-Rap1B and pGEX-4T-3-Rap2A mutants were generated using a Stratagene (La Jolla, CA, USA) QuickChange site-directed mutagenesis kit, and sequences were verified by sequencing.

\section{Protein expression and purification}

RasGEF1A, RasGEF1B, Rap1B mutants and Rap2A mutants were expressed at $23{ }^{\circ} \mathrm{C}$ and induced at a $A_{600} \mathrm{~nm}$ of 0.6 using $1 \mathrm{~mm}$ isopropyl thio- $\beta$-D-galactoside (IPTG) for 16 h. DiRas2, M-Ras, R-Ras, TC21, RalB, RERG, Rheb, ptac-H-Ras, ptac-Rap1A, ptac-Rap1B and ptacRap2A were expressed at $25^{\circ} \mathrm{C}$ after induction with $100 \mu \mathrm{M}$ IPTG at a $A_{600 \mathrm{~nm}}$ of 0.6 . Cell lysate supernatants of GST-fused RasGEF1A and RasGEF1B were applied to a glutathione (GSH) column (Amersham Biosciences, Freiburg, Germany) pre-equilibrated with buffer A [50 mM Tris ( $\mathrm{pH} 7.5$ ), $100 \mathrm{~mm} \mathrm{NaCl}, 3 \mathrm{~mm} \beta$-mercaptoethanol] at $4{ }^{\circ} \mathrm{C}$ and eluted with buffer A containing $20 \mathrm{~mm} \mathrm{GSH}$, and GST fusion proteins were purified by gel filtration on Superdex S75 16/60 columns (Amersham Biosciences). Cell lysate supernatants of the GST-fused G-proteins were applied to GSH Sepharose 4B (GE Healthcare, Chalfont St Giles) pre-equilibrated with buffer B [25 mM Tris (pH 7.5), $500 \mathrm{~mm} \mathrm{NaCl}, 5 \mathrm{~mm}$ dithioerythritol] at $4{ }^{\circ} \mathrm{C}$, and either eluted with $20 \mathrm{~mm}$ GSH and subsequently digested by tobacco etch virus protease in batches, or cleaved by thrombin on the column. Cleaved proteins were further purified by gel filtration and another GSH column to separate G-proteins from GST; ptac-cloned proteins were purified by Q-Sepharose and subsequent gel filtration as described previously [55]. Two milligrams from each protein was loaded with $N$-methylanthranil acid-labeled guanine nucleotide (mant-GNP) by incubating with $400 \mu \mathrm{M}$ $\mathrm{mGppNHp}$ and $1 \mathrm{U} \cdot \mathrm{mg}^{-1}$ alkaline phosphatase (Roche Diagnostics, Indianapolis, IN, USA) in an exchange buffer [200 mM (NH4) $\left.)_{2} \mathrm{SO}_{4}, 1 \mathrm{~mm} \quad \mathrm{ZnCl}_{2}\right]$ for $16 \mathrm{~h}$ at $4{ }^{\circ} \mathrm{C}$. Exchange of nucleotides was monitored by HPLC. After exchange, proteins were separated from free nucleosides and alkaline phosphatase by gel filtration (S75 10/30). Purified proteins were concentrated, flash-frozen in liquid nitrogen, and stored at $-80{ }^{\circ} \mathrm{C}$.

\section{Nucleotide exchange reaction}

Nucleotide exchange reactions were performed as described previously [56] in buffer C [50 mM Tris ( $\mathrm{pH} 7.5), 100 \mathrm{~mm}$ $\mathrm{NaCl}, 5 \mathrm{mM} \mathrm{MgCl}_{2}, 3 \mathrm{~mm} \beta$-mercaptoethanol] at $25^{\circ} \mathrm{C}$. G-proteins $(150 \mathrm{nM})$ were incubated with either $4 \mu \mathrm{M}$ or $200 \mathrm{~nm}$ RasGEF1s, and the reaction was started with $10 \mu \mathrm{M}$ GDP. The fluorescence change was detected at $450 \mathrm{~nm}$ after excitation at $366 \mathrm{~nm}$. With different preparations of RasGEF1A and RasGEF1B, nucleotide exchange rates varied by less than $30 \%$. Data were fitted to a single exponential equation using GRAFIT 5.0 (http://www. erithacus.com/grafit/).

\section{Acknowledgements}

E. Yaman was supported by mobility grants from TUBITAK-SBAG 105-S-365 and from the EU 7th Framework Programme Project UNAM-REGPOT (grant no. 203953). R. Gasper was supported by the International Max Planck Research School for Chemical Biology in Dortmund. This work was supported by the Turkish Academy of Sciences-Young Scientist Award (GEBIP/2001-2-18) and the Feyzi Akkaya Research Fund for Scientific Activities (FABED) to U. H. Tazebay.

\section{References}

1 Vetter IR \& Wittinghofer A (2001) The guanine nucleotide-binding switch in three dimensions. Science 294, 1299-1304. 
2 Bos JL, Rehmann H \& Wittinghofer A (2007) GEFs and GAPs: critical elements in the control of small G proteins. Cell 129, 865-877.

3 Chakrabarti PP, Daumke O, Suveyzdis Y, Kötting C, Gerwert K \& Wittinghofer A (2007) Insight into catalysis of a unique GTPase reaction by a combined biochemical and FTIR approach. J Mol Biol 367, 983-995.

4 McLeod SJ, Shum AJ, Lee RL, Takei F \& Gold MR (2004) The Rap GTPases regulate integrin-mediated adhesion, cell spreading, actin polymerization, and Pyk2 tyrosine phosphorylation in B lymphocytes. $J$ Biol Chem 279, 12009-12019.

5 Durand CA, Westendorf J, Tse KW \& Gold MR (2006) The Rap GTPases mediate CXCL13- and sphingosine1-phosphate-induced chemotaxis, adhesion, and Pyk2 tyrosine phosphorylation in B lymphocytes. Eur J Immun 36, 2235-2249.

6 Miertzschke M, Stanley P, Bunney TD, Rodrigues-Lima F, Hogg N \& Katan M (2007) Characterization of interactions of adapter protein RAPL/Norelb with Rap GTPases and their role in T cell migration. $J$ Biol Chem 282, 30629-30642.

7 Zhu Y, Pak D, Qin Y, McCormack SG, Kim MJ, Baumgart JP, Velamoor V, Auberson YP, Osten P \& van Aelst L (2005) Rap2-Jnk removes synaptic AMPA receptors during depotentiation. Neuron 46, 905-916.

8 Fu Z, Lee SH, Simonetta A, Hansen J, Sheng M \& Pak DT (2007) Differential roles of Rap1 and Rap2 small GTPases in neurite retraction and synapse elimination in hippocampal spiny neurons. $J$ Neurochem $\mathbf{1 0 0}$, 118-131.

9 Spilker C, Acuña Sanhueza GA, Böckers TM, Kreutz MR \& Gundelfinger ED (2008) SPAR2, a novel sparrelated protein with GAP activity for Rap1 and Rap2. $J$ Neurochem 104, 187-201.

10 Bos JL, de Rooij J \& Reedquist KA (2001) Rap1 signalling: adhering to new models. Nat Rev Mol Cell Biol 2, 369-377.

11 Nancy V, Wolthuis RM, de Tand MF, Janoueix-Lerosey I, Bos JL \& de Gunzburg J (1999) Identification and characterization of potential effector molecules of the Ras-related GTPase Rap2. J Biol Chem 274, 87378745.

12 Rodriguez-Viciana P, Sabatier C \& McCormick F (2004) Signaling specificity by Ras family GTPases is determined by the full spectrum of effectors they regulate. Mol Cell Biol 24, 4943-4954.

13 Wohlgemuth S, Kiel C, Krämer A, Serrano L, Wittinghofer F \& Herrmann C (2005) Recognizing and defining true Ras binding domains I: biochemical analysis. $J$ Mol Biol 348, 741-758.

14 Bunney TD, Harris R, Gandarillas NL, Josephs MB, Roe SM, Sorli SC, Paterson HF, Rodrigues-Lima F, Esposito D \& Ponting CP (2006) Structural and mechanistic insights into Ras association domains of Phospholipase-C-epsilon. Mol Cell 21, 495-507.

15 Zwartkruis FJ, Wolthuis RM, Nabben NM, Franke B \& Bos JL (1998) Extracellular signal-regulated activation of Rap1 fails to interfere in Ras effector signalling. EMBO J 17, 5905-5912.

16 Altschuler DL \& Ribeiro-Neto F (1998) Mitogenic and oncogenic properties of the small $\mathrm{G}$ protein Rap1b. Proc Natl Acad Sci USA 95, 7475-7479.

17 Posern G, Weber CK, Rapp UR \& Feller SM (1998) Activity of Rap1 is regulated by bombesin, cell adhesion, and cell density in NIH3T3 fibroblasts. $J$ Biol Chem 273, 24297-24300.

18 Asha H, de Ruiter ND, Wang MG \& Hariharan IK (1999) The Rap1 GTPase functions as a regulator of morphogenesis in vivo. EMBO J 18, 605-615.

19 Taira K, Umikawa M, Takei K, Myagmar BE, Shinzato M, Machida N, Uezato H, Nonaka S \& Kariya K (2004) The Traf2- and Nck-interacting kinase as a putative effector of Rap2 to regulate actin cytoskeleton. J Biol Chem 279, 49488-49496.

20 Paganini S, Guidetti GF, Catricalà S, Trionfini $\mathrm{P}$, Panelli S, Balduini C \& Torti M (2006) Identification and biochemical characterization of Rap2c, a new member of the Rap family of small GTP-binding proteins. Biochimie 88, 285-295.

21 McLeod SJ, Li AH, Lee RL, Burgess AE \& Gold MR (2002) The Rap GTPases regulate B cell migration toward the chemokine stromal cell-derived factor-1 (CXCL12): potential role for Rap2 in promoting B cell migration. J Immunol 169, 1365-1371.

22 Christian SL, Lee RL, McLeod SJ, Burgess AE, Li AH, Dang-Lawson M, Lin KB \& Gold MR (2003) Activation of the Rap GTPases in B lymphocytes modulates $\mathrm{B}$ cell antigen receptor-induced activation of Akt but has no effect on MAPK activation. J Biol Chem 278, 41756-41767.

23 Huang CC, You JL, Wu MY \& Hsu KS (2004) Rap1Induced p38 mitogen-activated protein kinase activation facilitates AMPA receptor trafficking via the GDI.Rab5 complex. Potential role in (S)-3,5-dihydroxyphenylglycene-induced long term depression. J Biol Chem 279, 12286-12292.

24 Imamura Y, Matsumoto N, Kondo S, Kitayama H \& Noda M (2003) Possible involvement of Rap1 and Ras in glutamatergic synaptic transmission. Neuroreport 14, 1203-1207.

25 Machida N, Umikawa M, Takei K, Sakima N, Myagmar BE, Taira K, Uezato H, Ogawa Y \& Kariya K (2004) Mitogen-activated protein kinase kinase kinase kinase 4 as a putative effector of Rap2 to activate the c-Jun n-terminal kinase. J Biol Chem 279, 15711-15714.

26 Kukimoto-Niino M, Takagi T, Akasaka R, Murayama K, Uchikubo-Kamo T, Terada T, Inoue M, Watanabe S, Tanaka A \& Hayashizaki Y (2006) Crystal structure 
of the RUN domain of the Rap2-interacting protein. $J$ Biol Chem 281, 31843-31853.

27 Rehmann H, Arias-Palomo E, Hadders MA, Schwede F, Llorca O \& Bos JL (2008) Structure of Epac2 in complex with a cyclic AMP analogue and Rap1B. Nature 455, 124-127.

28 Boriack-Sjodin PA, Margarit SM, Bar-Sagi D \& Kuriyan J (1998) The structural basis of the activation of Ras by Sos. Nature 394, 337-343.

29 Béranger F, Goud B, Tavitian A \& de Gunzburg J (1991) Association of the Ras-antagonistic Rap1/Krev-1 proteins with the Golgi complex. Proc Natl Acad Sci USA 88, 1606-1610.

30 Béranger F, Tavitian A \& de Gunzburg J (1991) Posttranslational processing and subcellular localization of the Ras-related Rap2 protein. Oncogene 6, 1835-1842.

31 Pizon V, Desjardins M, Bucci C, Parton RG \& Zerial M (1994) Association of Rapla and Raplb proteins with late endocytic/phagocytic compartments and Rap2a with the Golgi complex. J Cell Sci 107, 16611670.

32 Pannekoek WJ, Kooistra MR, Zwartkruis FJ \& Bos JL (2008) Cell-cell junction formation: the role of Rap1 and Rap1 guanine nucleotide exchange factors. Biochim Biophys Acta 1788, 790-796.

33 van den Berghe N, Cool RH \& Wittinghofer A (1999) Discriminatory residues in Ras and Rap for guanine nucleotide exchange factor recognition. $J$ Biol Chem 274, 11078-11085.

34 de Rooij J, Rehmann H, van Triest M, Cool RH, Wittinghofer A \& Bos JL (2000) Mechanism of regulation of the Epac family of cAMP-dependent RapGEFs. J Biol Chem 275, 20829-20836.

35 Bos JL (2006) Epac proteins: multi-Purpose cAMP targets. Trends Biochem Sci 31, 680-686.

36 de Rooij J, Boenink NM, van Triest M, Cool RH, Wittinghofer A \& Bos JL (1999) PDZ-GEF1, a guanine nucleotide exchange factor specific for Rap1 and Rap2. $J$ Biol Chem 274, 38125-38130.

37 Ebinu JO, Bottorff DA, Chan EY, Stang SL, Dunn RJ \& Stone JC (1998) RasGRP, a Ras guanyl nucleotidereleasing protein with calcium- and diacylglycerol-binding motifs. Science 280, 1082-1086.

38 Bivona TG, Pérez De Castro I, Ahearn IM, Grana TM, Chiu VK, Lockyer PJ, Cullen PJ, Pellicer A, Cox AD \& Philips MR (2003) Phospholipase-c-gamma activates Ras on the Golgi apparatus by means of RasGRP1. Nature 424, 694-698.

39 Lenzen C, Cool RH \& Wittinghofer A (1995) Analysis of intrinsic and Cdc25-stimulated guanine nucleotide exchange of p21Ras-nucleotide complexes by fluorescence measurements. Methods Enzymol 255, 95-109.

40 Klebe C, Prinz H, Wittinghofer A \& Goody RS (1995) The kinetic mechanism of Ran-nucleotide exchange catalyzed by RCC1. Biochemistry 34, 12543-12552.
41 Esters H, Alexandrov K, Iakovenko A, Ivanova T, Thomä N, Rybin V, Zerial M, Scheidig AJ \& Goody RS (2001) Vps9, Rabex-5 and DSS4: proteins with weak but distinct nucleotide-exchange activities for Rab proteins. J Mol Biol 319, 141-156.

42 Takai Y, Sasaki T \& Matozaki T (2001) Small GTPbinding proteins. Physiol Rev 81, 153-208.

43 Colicelli J (2004) Human RAS superfamily proteins and related GTPases. STKE 2004 (250), RE13.

44 Karnoub AE \& Weinberg RA (2008) Ras oncogenes: split personalities. Nat Rev Mol Cell Biol 9, 517-531.

45 Mistou MY, Jacquet E, Poullet P, Rensland H, Gideon P, Schlichting I, Wittinghofer A \& Parmeggiani A (1992) Mutations of Ha-Ras p21 that define important regions for the molecular mechanism of the SDC25 c-domain, a guanine nucleotide dissociation stimulator. EMBO J 11, 2391-2397.

46 Mosteller RD, Park W \& Broek D (1995) Analysis of interaction between Ras and CDC25 guanine nucleotide exchange factor using yeast GAL4 two-hybrid system. Methods Enzymol 255, 135-148.

47 Quilliam LA, Hisaka MM, Zhong S, Lowry A, Mosteller RD, Han J, Drugan JK, Broek D, Campbell SL \& Der CJ (1996) Involvement of the switch 2 domain of Ras in its interaction with guanine nucleotide exchange factors. J Biol Chem 271, 11076-11082.

48 Schmidt M, Evellin S, Weernink PA, von Dorp F, Rehmann H, Lomasney JW \& Jakobs KH (2001) A new phospholipase-c-calcium signalling pathway mediated by cyclic AMP and a Rap GTPase. Nat Cell Biol 3, 1020-1024.

49 Choi SC \& Han JK (2005) Rap2 is required for Wnt/beta-catenin signaling pathway in Xenopus early development. EMBO J 24, 985-996.

50 Greco F, Ciana A, Pietra D, Balduini C, Minetti G \& Torti M (2006) Rap2, but not Rap1 GTPase is expressed in human red blood cells and is involved in vesiculation. Biochim Biophys Acta 1763, 330-335.

51 Gasper R, Thomas C, Ahmadian MR \& Wittinghofer A (2008) The role of the conserved switch II glutamate in guanine nucleotide exchange factor-mediated nucleotide exchange of GTP-binding proteins. J Mol Biol 379, $51-63$.

52 Ura K, Obama K, Satoh S, Sakai Y, Nakamura Y \& Furukawa Y (2006) Enhanced RASGEF1A expression is involved in the growth and migration of intrahepatic cholangiocarcinoma. Clin Cancer 12, 6611-6616.

53 Ferreira LRP, Abrantes EF, Rodrigues CV, Caetano B, Cerqueria CG, Christina-Salim A, Reis LFL \& Gazzinelli RT (2002) Identification and characterization of a novel mouse gene encoding a Ras-associated guanine nucleotide exchange factor: expression in macrophages and myocarditis elicited by Trypanosoma cruzi parasites. J Leukoc Biol 72, 1215-1227. 
54 Epting D, Vorwerk S, Hageman A \& Meyer D (2007) Expression of RasGEF1b in Zebrafish. Gene Express Patterns 7, 389-395.

55 van den Berghe N, Cool RH, Horn G \& Wittinghofer A (1997) Biochemical characterization of C3G: an exchange factor that discriminates between Rap1 and Rap2 and is not inhibited by Rapla(S17N). Oncogene 15, 845-850.

56 Tucker J, Sczakiel G, Feuerstein J, John J, Goody RS \& Wittinghofer A (1986) Expression of p21 proteins in Escherichia coli and stereochemistry of the nucleotidebinding site. EMBO J 5, 1351-1358.

57 Marti-Renom MA, Stuart AC, Fiser A, Sanchez R, Melo F \& Sali A (2000) Comparative protein structure modeling of genes and genomes. Annu Rev Biophys Biomol Struct 29, 291-325.

58 Emsley P \& Cowtan K (2004) Coot: Model building tools for molecular graphics. Acta Crystallogr D Biol Crystallogr 60, 2126-2132.

\section{Supporting information}

The following supplementary material is available:

Fig. S1. Guanine nucleotide exchange reaction of $150 \mathrm{~nm}$ Rap1B(S39F) with increasing concentrations of RasGEF1A.

Table S1. Rate constants of mGNP release with RasGEF1A and RasGEF1B.

This supplementary material can be found in the online article.

Please note: As a service to our authors and readers, this journal provides supporting information supplied by the authors. Such materials are peer-reviewed and may be re-organized for online delivery, but are not copy-edited or typeset. Technical support issues arising from supporting information (other than missing files) should be addressed to the authors. 